\title{
ESTILO DE VIDA SALUDABLE (EVS): LIMITACIONES DEL ENFOQUE BIOMÉDICO
}

\section{Daniel Pinzón Gómez}

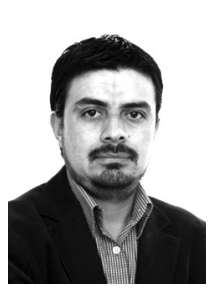

Administrador en salud (Universidad de Antioquia, Colombia). Candidato a la Maestría en Salud Pública (Atlantic International University, EE.UU). Docente investigador de la Corporación Universitaria Adventista (UNAC), Colombia, en el área de atención prehospitalaria y de estilo de vida saludable. Coinvestigador del grupo de epidemiología de la Facultad Nacional de Salud Pública (FNSP) de la Universidad de Antioquia, Colombia, en la línea de trauma. Ha liderado proyectos relacionados con gestión del riesgo de desastres, sistemas de emergencias y seguridad. $\mathrm{Ha}$ participado en investigación de la situación de los sistemas de servicios de emergencias médicas de Latinoamérica y el Caribe (OPS - FNSP). Ha apoyado, asimismo, en programas humanitarios en desastres para Colombia y Haití. 


\section{Resumen}

Este artículo plantea las limitaciones que ofrecen los estilos de vida saludables, en el discurso de la epidemiología clásica, que centra su argumento en la conducta individual de las personas; se discute el énfasis de la investigación en estilos de vida saludables que realiza la Universidad de Loma Linda desde 1958 en adventistas y la carencia de estudios en esta población aleatorizados en países periféricos; plantea la necesidad de analizar las restricciones del estilo de vida saludable adventista, desde las aportaciones de la epidemiología social, particularmente en el contexto de los procesos de determinación social de la salud.

Palabras clave: Estilo de vida saludable, adventistas, biomédico, epidemiología social, salud pública, determinantes sociales.

\section{Abstract}

This article remarks some limitations that healthy lifestyles offer to biomedical speech, which centers its argument on individual behavior of people; it also emphasizes investigations in Adventists of developed countries and the lack of randomized studies with this population in outlying countries. At the same time, it establishes the necessity to analyze the restrictions of healthy lifestyle, taking into account the contributions of social epidemiology, particularly in the context of social determinants. Finally, it mentions the importance of reducing inequity in health, as well as the necessity of theoretical contributions from other disciplines to understand health and its connection with healthy lifestyles beyond the biomedical focus.

Keywords: Healthy lifestyles, adventist church, biomedic, social epidemiology, public health, social determinants. 


\section{Introducción}

La comunidad científica adventista continuamente encuentra evidencia que respalda la filosofía de la salud, particularmente, en lo que se refiere a los Estilos de Vida Saludables (EVS), dado que está asociado a una larga longevidad (9,4 años) y menor riesgo de padecer enfermedades crónicas no transmisibles (ECNT) (Álvarez, 2012; Cerecero et al, 2009; Friis, 2009; Fall, 2010; Fraser, 2003). Pero ¿qué se conoce de la evidencia e investigación científica que identifica limitaciones en este enfoque?

Desde que la noción de estilo de vida fue tomada de las ciencias sociales e incorporada al acervo de la epidemiología clásica (Álvarez, 2012), bajo el concepto de estilo de vida saludable (EVS) en los años sesenta (Vargas, 2010), las referencias que tratan sobre el particular han crecido de manera sostenida con un significado, dirigido a la conducta de los individuos (Cockerham, 2012); de igual manera, investigaciones ponen de manifiesto las limitaciones de este enfoque, dado su carácter restringido a la conducta individual.

Este artículo expone las restricciones del enfoque de EVS en lo que respecta a alimentación, consumo de agua, respiración de aire puro, actividad física y descanso, entre otros, principios ampliamente divulgados por los Adventist Health Studies (AHS) y liderados por las instituciones de salud adscritos a la Iglesia Adventista del Séptimo Día, principalmente por la Universidad de Loma Linda de Estados Unidos y otros estudios realizados en población adventista por agencias e investigadores particulares. No obstante, pretende hacer a una reflexión crítica, más allá de la argumentación biomédica y analizar de qué manera el EVS puede estar condicionado igualmente por procesos de determinación social, marginalmente considerados por la epidemiología clásica.

\section{Interpretación del proceso salud - enfermedad y la salud de los adventistas}

Desde la perspectiva científica, la interpretación del proceso salud - enfermedad se puede categorizar desde dos vertientes: aproximaciones medicalizadas y holísticas ${ }^{1}$ (Gómez, 2002).

Las primeras están centradas en la enfermedad como fenómeno

\footnotetext{
${ }^{1}$ El término holístico significa, según el diccionario de la lengua española (C) 2005 Espasa-Calpe: "Del todo o relativo a él”. El término holístico (holismo) se entiende en la teoría general de los sistemas (TGS) como que las cosas (sistemas naturales y sociales) deben ser entendidos como un todo y no de forma separada. Así, en el contexto de este artículo se refiere a que la salud debe entenderse como un constructo que trasciende la interpretación de la clínica y la epidemiología clásica, más allá de las partes separadas o reduccionistas que ha propuesto las ciencias médicas. El término tiende a usarse más ampliamente en el lenguaje académico y
} 
individual, altamente influenciadas por la biología y sus ciencias subsidiarias, cuya principal herramienta es la clínica, lo cual queda plenamente demostrado en el modelo de educación flexneriano común en las escuelas de medicina de Occidente (Bosch, 2006). Este enfoque pone énfasis en el reduccionismo científico de la vida a través del racionamiento científico positivista (empíricoanalítico $)^{2}$. Las segundas cada vez más cercanas a la noción de calidad de vida, reconocen al individuo como un ser biológico, psicológico, social y espiritual desde un razonamiento científico histórico - hermenéutico y crítico - social apoyadas por disciplinas y ciencias con carácter interdisciplinarios, incluso transdisciplinarios. De esta manera la búsqueda de la salud se dispersa en torno a la vida en el complejo sistema determinado por el funcionamiento encadenado y sinérgico de la organización biológica, ecológica, social y su conexión con lo divino (célula, órgano, individuo, familia, comunidad, población, sociedad, ecosistema y el cosmos).

La filosofía adventista reconoce la composición bio-psico-socioespiritual (Fuentes, 2012) del ser humano desde la perspectiva teísta y que tal posición es legitimada por la tradicional definición de salud propuesta por la Organización Mundial de la Salud (OMS), en 1948, que incluye los componentes biológico, psicológico y social. Recientemente este órgano ha incluido la dimensión espiritual como elemento esencial de la salud y ha recomendado la modificación del concepto clásico, considerando que "la salud es un estado de completo bienestar físico, mental, social y espiritual y no solamente la ausencia de dolencia o enfermedad". Hasta el momento tal modificación no se ha producido (Jimenez, 2012).

Uno de los paradigmas para entender los procesos relacionados con la construcción científica de la filosofía de la salud adventista, lo constituye la teoría de "campos de salud" desarrollada por Hubert Laframboise en 1973 e impulsada magistralmente por Lalonde (1974), y que es conocido como el Lalonde Report o White Paper, la cual, entre otros aspectos pondera la participación de los determinantes de la salud en el seno de la sociedad canadiense y que ha sido

científico en sustitución de la tradicional acepción "integral". El término es usado ampliamente por la literatura científica y especialmente en publicaciones de salud. En este sentido, se precisa que el término carece de connotación esotérica.

${ }^{2}$ El positivismo es la corriente filosófica que nace con F. Bacon $(1561$ - 1678) y defendida posteriormente por Auguste Comte (1798 - 1857) y John Stuart Mill (1806 - 1873) en la que está fundamentada la investigación cuantitativa de hoy, su planteamiento más concreto se recoge en el postulado de Galileo Galilei: "Mide lo que sea medible y haz medible lo que no lo sea". El positivismo científico es una de las corrientes que ha descartado la existencia de una deidad, por su carácter empirista y cuantificable de su objeto de estudio. Paradójicamente el método positivista es el más usado en las publicaciones e investigaciones científicas de la salud adventista. Cfr. los métodos epidemiológicos en el estudio de salud adventista realizados por la Universidad de Loma Linda, en los cuales el lector puede identificar el énfasis positivista en los instrumentos de investigación. 
recogido por varios sistemas de salud del mundo, especialmente por los que justifican las teorías neoliberales del mercado sanitario.

La mayoría de las corrientes y modelos de la medicina preventiva, salud pública y en países desarrollados se acogen a este modelo y los que se han desprendido de este y otros estudios (Dever, 1976; entre otros). Pese a los 40 años de su existencia y partiendo de la realidad concreta canadiense, es una doctrina invariable en las escuelas de salud de los países en vías de desarrollo que se presume extrapolable en estas sociedades (Álvarez et al, 2007). Este modelo estimula la idea que los estilos de vida es la variable que más aporta a la carga de morbilidad, mortalidad y al gasto en salud, que representa el rubro con menos recursos por parte del sistema sanitario (Vargas, 2010; Lalonde, 1974; Álvarez et al, 2007; Dever, 1976).

El enfoque de EVS (Álvarez et al, 2007; Dever, 1976 y Cerecero et al, 2009), ha demostrado de manera exitosa que existe una relación directa entre el estrés, sedentarismo, sobrepeso, consumo de alcohol, tabaco y el alto consumo de grasas con enfermedades crónicas, especialmente asociadas al riesgo cardiovascular y diabetes tipo 2 . El modelo hace énfasis en que la salud es una responsabilidad individual.

De la misma manera, en el contexto de la interpretación adventista, la salud es considerada como una responsabilidad individual, que se materializa en hábitos saludables; entiende también que la enfermedad está relacionada con inadecuados hábitos en el comer y, en general, en la forma cómo conducen sus vidas las personas, por lo cual corresponde al individuo asumir su compromiso de cuidarla (Gálvez, 2002; Nieman, 1992). Aunque las directrices sanitarias que fueron impartidas por Yahvé, especialmente en el Pentateuco ${ }^{3}$ a la familia edénica y por extensión a los hebreos, están enmarcadas en una estructura de salud pública y colectiva, la filosofía de la salud adventista presenta un énfasis en los aspectos comportamentales del individuo, los cuales fueron discutidos y ampliados por White (2010) a fines del siglo XIX.

El estilo de vida adventista ha sido continuamente legitimado por la evidencia científica. La observancia de los Ochos Remedios Naturales (consumo de agua, sueño apropiado, dieta vegetariana, actividad física, toma de sol moderada, aire puro, temperancia y desarrollo de una vida espiritual) (White, 2010; Asociación General de los Adventistas del Séptimo Día, 2006; Scheneider,

${ }^{3}$ Santa Biblia. Versión Reina Valera. Brasil. Sociedades Bíblicas Unidas; 1995. Génesis 1: 28, 29.Precepción de una dieta vegetariana para la familia edénica. Éxodo 20: 1- 17. Los 10 mandamientos. Particularmente la observancia del sábado (cuarto mandamiento) es considerada una medida de salud pública. El mandamiento sexto "No matarás" es igualmente interpretado como una medida de salud pública que tiene implicaciones personales y colectivas. Levítico 7:23,24. Restricciones frente al consumo de grasa animal. Levítico 11. Leyes dietéticas. Lev. 17:10 - 16. Prohibición de comer sangre. Deuteronomio 23:9-14. Leyes sanitarias. 
2004; Pamplona, 2004), incide de manera significativa en una mayor longevidad entre adventistas y la reducción de padecer las enfermedades crónicas no transmisibles (ECNT). Estudios de cohorte prospectivos realizados por la Universidad de Loma Linda evidencian las bondades de un EVS ${ }^{4}$ (Pamplona, 2004), sobre los adventistas de California (Fuentes, 2012; Nieman, 1992; Buettner, 2005; Beeson et al, 1989; Mills, et al, 1989; Beeson, 1999; Fonnebo y Helseth, 1961 y Buettner, 2008). Justamente, los estudios liderados por Buettner (2005, 2008; Appel, 2008), permiten ubicar al grupo más longevo y saludable de los EE.UU. y de las Américas, los adventistas residentes en el municipio de Loma Linda, California, una de las Blue Zones del mundo (Buettner, 2008). Estudios similares en adventistas (Fuentes, 2012; Nieman 1992; Beeson, 1999) en Noruega (Guerrero et al 2010), Dinamarca (Jenson, 1993), Polonia, Holanda (Berkel y deWaard, 1983), Japón (Kuratsune et al, 1986), Australia han arrojado resultados simulares.

\section{Antecedentes sobre el concepto estilo de vida saludable}

El concepto de estilo de vida nace en las ciencias sociales (Álvarez, 2012; Vargas, 2010; Cockerham, 2012; Fuentes, 2012), siendo Karl Marx y Max Weber (Albert et al, 2002; OMS, 2010; De la Antonia, 2009), quienes ofrecieran los primeros aportes sobre esta acepción. Estos autores brindan una visión sociológica del estilo de vida, enfatizando los determinantes sociales de su adopción y mantenimiento. No obstante es Marx Weber el mayor contribuyente en la construcción de estilo de vida y estilo de vida saludable. Desde su teoría de la racionalidad formal y las ideas acerca del estilo de vida, posibilitan el inicio para que otros autores apliquen las ideas propuestas por él en el desarrollo del concepto sobre estilo de vida en salud (Vargas, 2010; Guerrero et al, 2010). Weber relacionó que el estilo de vida (Lebensstil) de las personas está dado por la conducta de vida (Lebensfübrung) y estas a su vez están condicionadas por las oportunidades de vida (Lebenchancen), considerando que las oportunidades de vida finalmente están determinadas por las circunstancias sociales, específicamente las socioeconómicas (Vargas, 2010; Cockerham, 2012). De esta manera este autor desarrolló un concepto holístico de estilo de vida fundamentado en aspectos como ocupación, el ingreso, la educación y el estatus, logrando formular posteriormente el nivel

${ }^{4}$ Desde 1958 la Escuela de Salud Pública de la Universidad de Loma Linda, California, Estados Unidos, ha liderado el estudio de salud adventista (Adventist Health Study) realizando investigaciones relacionadas con el estilo de vida de los adventistas, con la mortalidad y con la prevalencia de enfermedades crónicas no transmisibles como el cáncer y la enfermedad isquémica del corazón. Información sobre este estudio puede ser consultada en la página: www.llu.edu. 
socioeconómico (Cockerham, 2012).

Las corrientes de la epidemiología clásica comenzaron la adopción del término estilos de vida y salud desde las décadas de 1950 y 1960, logrando tener su máximo apogeo en la década de los ochenta (Vargas, 2010), momento en el que se presenta el mayor desarrollo investigativo en la materia, siendo usados los términos estilos de vida, estilos de vida en salud y estilo de vida saludable de manera indistinta y entendiéndose como sinónimos (Álvarez, 2012; Vargas, 2010), para referirse a la conducta individual vinculada con las ECNT, exclusivamente, salvo el VIH - SIDA. En tiempos más recientes, el término fue llevado por la corriente más positivista de la epidemiología a la noción riesgo (factores de riesgo) y causalidad, acentuando la responsabilidad de los individuos en su salud (Álvarez, 2012) y alejándose de las interpretaciones originales identificadas por las ciencias sociales (De Almeida, 2000; Breilh, 2003; Menéndez, 1998). Pese a que en el discurso y la práctica la epidemiología clásica y la medicina preventiva le siguen dando un énfasis restringido a la conducta individual y a la elección voluntaria de las personas, la OMS define el estilo de vida "como una forma general de vida basada en patrones de comportamiento identificables, determinados por la interacción entre características personales individuales, las interacciones sociales y las condiciones de vida socioeconómicas y ambientales" (Vargas, 2010; Fernández et al, 2012; Guerrero et al, 2010). De esta manera el órgano multilateral de salud reconoce en la definición que el comportamiento humano asume estructuras complejas, el cual se desarrolla y se manifiesta en condiciones sociales, económicas, ecológicas y culturales específicas y en el proceso de socialización de los individuos emergen las experiencias de su historia que inciden en su comportamiento $\mathrm{y}$, desde luego, en su situación de salud (Hall, 2010).

\section{Limitaciones del enfoque de estilos de vida saludable}

La noción de EVS, que ha tenido mucha fuerza en las últimas cuatro décadas, también ha sido acompañada por fuertes críticas desde su aparición, entre las que se describen a continuación:

1. Los sujetos pueden y desean cambiar sus hábitos. Al centrarse en las conductas y características de los individuos, en el enfoque de EVS se parte de la premisa que los sujetos pueden y desean cambiar sus conductas, con lo cual frecuentemente se desconocen los contextos sociales, económicos, políticos y culturales en las que se pudieran dar estas prácticas (Breilh, 
2003; Guerrero et al, 2010; Ortiz, 2007). Dos ejemplos en la experiencia de Latinoamérica y el Caribe (LAC) recrean lo dicho: Haití y Colombia. Para el primero, las condiciones de pobreza material es el principal determinante económico que impide que esta población adopte el EVS, incluso entre adventistas institucionalizados 5 . Esta es una situación que en las indagaciones efectuadas a investigadores de AHS de la Universidad de Loma Linda manifiestan no estar consideradas.

Para el caso de Colombia que, a diferencia de Haití, es un país en transición al desarrollo, los conflictos armados, el problema narco, el daño ambiental por deforestación y aspersiones en cultivos ilícitos y los desplazamientos forzados tienen un gran impacto en la salud pública y los derechos humanos que (por lo general afectan a las personas más desfavorecidas) agudizan y empeoran las condiciones de pobreza en las que ya están inmersas las poblaciones, limitando la posibilidad de escoger libremente alimentos saludables, consumir agua (en algunas condiciones limitada o no potable) hacer ejercicio, dormir tranquila y adecuadamente, entre otras prácticas. De esta manera los comportamientos y conductas derivados de una situación de estrés psicosocial por pobrezas crónicas no es el resultado de decisiones o acciones voluntarias individuales, es el producto de desajustes sociales, por lo cual resultaría inocuo hacer recomendaciones orientadas a cambiar comportamientos a menos que esas condiciones macrosociales cambien. No parece razonable recomendar a una persona que padece hambre crónico y que limite su ingesta de alimentos con alto contenido de grasa, cuando es su única fuente para saciar el hambre. No sería prudente indicarle a una persona que duerma ocho (8) horas durante la noche, cuando está sometida a una constante presión psicosocial de actores armados.

Para algunos críticos, los ejemplos citados pueden considerarse situaciones extremas y no generalizadas para comunidades de LAC, no obstante, las condiciones socioeconómicas representadas en acceso a alimentos, nivel de pobreza, empleos, condiciones laborales y las necesidades básicas insatisfechas (NBI), así como las condiciones inadecuadas de trabajo vulneran las posibilidades de una calidad de vida integral (Breilh, 2003) y el desarrollo de un estilo de vida salubérrimo y sostenible (Vargas, 2010). Las

${ }^{5}$ Durante la fase posdesastre del terremoto de Haití en el 2010, el autor evidenció en las instituciones adventistas la provisión de alimentos con proteína de origen animal, en virtud de la inseguridad alimentaria generalizada en la isla. 
condiciones de pobreza, marginalización e inequidad presentan relaciones directas con la localización geográfica, las condiciones sociodemográficas y el acceso a los alimentos (seguridad alimentaria) (Álvarez-Uribe y EstradaRestrepo, 2008). Por lo cual, las acciones de promoción de la salud deben dar prioridad a focalizar los esfuerzos para la reducción de la pobreza, el hambre y la desnutrición en los hogares, antes que a las acciones educativas orientadas al cambio de comportamientos.

2. La salud es responsabilidad individual. La concepción de los EVS se basa en la premisa que el mantenimiento de la salud y la prevención de la enfermedad es responsabilidad de los individuos y que estos pueden cambiar libre y voluntariamente sus conductas, con lo cual se omite la influencia de la organización de la sociedad y especialmente del mercado sobre el comportamiento de los individuos (Alvarez, 2012; Cockerham, 2012; Ortiz, 2007). Algunos Estados promueven los EVS para reducir el gasto en salud (Jiménez, 2012; Lalonde, 1974; Fernández et al, 2012 y Alcaldía de Medellín, 2012), sin embargo, promueven y/o fomentan las prácticas insalubres (a través del mercado) como el consumo de alcohol, tabaco y las drogas psicoactivas (Colombia ha despenalizado el porte y consumo de dosis mínima de marihuana y cocaína ${ }^{6}$ ) en aras de la defensa de las libertades individuales, de esta manera, igualmente se promueven el consumo de alimentos altamente energéticos, las plazas de comidas en espacios comerciales son un ejemplo de la promoción de estilos de vida insalubres, donde las opciones de encontrar alimentos saludables son limitadas. Todas estas prácticas son, a su vez, promovidas través de los medios de comunicación. Como hecho paradójico, hasta donde el autor conoce, ninguna escuela o facultad de salud en Colombia tiene una oferta de alimentos saludables (frutas, verduras y alimentos bajos en grasas) a través de sus cafeterías y restaurantes.

De otro lado, la ideología de hacer responsable al individuo por su salud y en caso de enfermar atribuirle el desenlace a responsabilidad (culpabilizar a la víctima) (Crawford, 1977), se constituye en uno de los instrumentos ideológicos y políticos para defender los sistemas de salud basados en el pluralismo estructurado7 (Frenk y Londoño, 1998) y el mercado del

${ }^{6}$ El Congreso. República de Colombia. Ley de Seguridad ciudadana. Ley 1453 de 2011.

${ }^{7}$ El pluralismo estructurado es el modelo político económico, formulado por el Banco Mundial, cuyos ideólogos fueron los doctores Julio Frenk (México) y Juan Luis Londoño (Colombia) para la implantación de modelos de salud neoliberales en Latinoamérica y el Caribe (LAC) en los que se insta a los Estados a abandonar la ideas bienestaristas de la salud como derecho público fundamental para convertirse en un servicio privado 
aseguramiento sanitario. Así, el problema es el comportamiento de los individuos frente al riesgo cuya solución es el cambio a voluntad a un EVS que se da a través de la educación o las sanciones culpabilizantes (culpabilizar a la víctima) (Crawford, 1977). Esta iniciativa políticoeconómica surge como ideología, justifica el recorte o la supresión del acceso a los servicios de salud como derecho fundamental e intenta, por otro lado, desviar la atención de la responsabilidad social en la aparición de las enfermedades que se derivan de las actividades propias de la globalización, sobre todo aquellas que tienen relación con la salud ambiental y ocupacional (Martínez, 2002). De esta manera, se entiende que la responsabilidad de la salud va más allá de la responsabilidad individual, requiere entenderse como un compromiso compartido entre el individuo, la sociedad y el Estado.

3. El enfoque de EVS privilegia los factores biológicos que predisponen a la enfermedad (Ortiz, citado por Jarillo y Guinsberg, 2007)). Para el caso de la obesidad, por ejemplo, se recurre a estudiar los determinantes genéticos y fisiológicos (Del Barrio, 2002), por ejemplo, en la obesidad, las investigaciones se centran en estudios moleculares (gen $\mathrm{Ob}$, la proteína leptina, el neuropéptido $Y)^{8}$; no obstante, se desconoce que los determinantes ambientales y psicosociales promueven o inhiben genes, por lo que el incremento de la frecuencia de la obesidad no puede ser explicada únicamente por la expresión génica en sí, sino por los cambios en el entorno y el ecosistema, los cuales muchas veces dependen de las condiciones sociales y económicas. El enfoque de los grandes problemas de salud (como lo puede ser la obesidad) se está centrando en factores intrínsecos a las personas, como sus genes y su comportamiento individual, mientras que el papel de los determinantes sociales es omitido (Del Barrio, 2002; Keyeux, 2007).

4. Las estrategias de educación en salud basadas en EVS (Ortiz, 2007). Las estrategias de prevención y tratamiento que han tomado como base el enfoque de EVS ha tenido una eficiencia limitada. Los estudios etnográficos ponen en evidencia que las personas que conocen los factores de riesgo, asociados a enfermedades crónicas, reconocen las dificultades

sometido a las fuerzas del mercado. Aunque Chile es el primer país en asumir la privatización de la salud en LAC, motivado por una dictadura en los años setenta, es Colombia quien lidera el proceso en la implementación del pluralismo estructurado en el marco de una democracia y que se materializó a través de la Ley 100 de 1993 [Cited 2012, August, 22]. Available from: http://www.causasdelaobesidad.com/cat/causas-de-la-obesidad/1 2012. 
para acogerse a una estrategia de EVS, debido a las limitaciones que se enfrentan cotidianamente, tales como el acceso a tiendas con variedad (las tiendas de barrios de bajos y medios ingresos no disponen de alimentos saludables para la venta) y calidad de artículos saludables, precios, condiciones de desplazamiento, (algunas personas, por razones de tiempo e ingresos, no pueden desplazarse a grandes superficies o a hipermercados para adquirir alimentos saludables), entre otras (Martínez, 2002).

Ante las limitaciones del enfoque de EVS, que si bien ha significado aportes sustantivos al mejoramiento de la calidad de vida de personas de condiciones socioeconómicas, educadas y con fácil acceso a una canasta familiar equilibrada, es necesario defender un paradigma más holístico, ético y moralmente acorde con las necesidades de los menos favorecidos (los pobres). La epidemiología social ofrece una aproximación a un enfoque más integral, la cual intenta entender cómo los problemas de alimentación y salud se relacionan con procesos sociales, culturales, políticos y económicos (World Health Organization, 2010; Dahlgren y Whitehead, 1991; Barton y Grant, 2012; 2006). Esta ciencia, a partir de un enfoque sociomédico, interpreta la desigualdad social en salud como una de las causales de enfermedad, la cual lo considera como un "acto inmoral e injusto" innecesario no inevitable ni irremediable (Guerrero, 2010), que exige un cambio en el paradigma y las formas de organización de la sociedad.

La organización adventista debería asumir una postura más crítica en aras de defender la filosofía que, aunque es bien intencionada y noble, presenta áreas sensibles y que, al defenderlas, deberían señalar de manera responsable los alcances y limitaciones que los EVS tienen, de otra forma se acrecienta la culpa de quienes, por razones económicas (y no espirituales), se les imposibilita estar en regla con un estilo de vida, en concordancia con la pauta denominacional.

De acuerdo a lo expuesto, la principal limitación que comportaría el enfoque de EVS para los miembros adventistas, en lo que concierne a dieta, consumo de agua, respiración de aire puro, actividad física y descanso adecuado, excluyendo el consumo de sustancias (alcohol, café, tabaco y drogas psicoactivas), es que difícilmente pudiera ser extrapolable a la mayoría de personas de esta comunidad, en virtud a sus limitaciones socioeconómicas. La mayoría de los adventistas se concentra en regiones del mundo con renta baja (89\%), (contra un $7 \%$ de Norteamérica y $4 \%$ que lo comparte Europa y Oceanía). Los adventistas se ubican en las regiones del mundo donde existen los mayores niveles de inequidad, desastres y guerras. Así, considerando que la mayor cantidad de estos adventistas se ubica en África (37\%), donde prevalecen 
las condiciones más extremas de hambre y pobreza crónicas, Latinoamérica y el Caribe (33\%) y Asia con un $19 \%$ de presencia adventista ${ }^{9}$ es posible inferir de estas cifras que la mayoría de los adventistas en el mundo son pobres, sin determinar cómo de distribuyen por clases sociales dentro de sus países y dentro de sus congregaciones.

Los estudios científicos que demuestran las ventajas del enfoque de estilo de vida adventista se han concentrado en países de renta alta como Estados Unidos, Canadá, Noruega, Dinamarca, Holanda, Polonia, Australia y Japón (Bosch, 2006; Gálvez, 2002; Schneider, 2004; Pamplona, 2004; Buettner, 2005; Beeson et al, 1989; Mills, et al, 1989; Beeson, 1999), algunos de estos estudios han tenido énfasis en población blanca no hispana adventista residentes en la localidad de Loma Linda, California, Estados Unidos, una de las comunidades más solventes de ese país, con ingresos medios por hogar de $\$ 38.204$ dólares, con una tradicional formación en salud y ciencias médicas ${ }^{10}$, condiciones sociales y económicas muy alejadas de la realidad de los países en desarrollo.

\section{Conclusión y recomendaciones}

Dado que en este análisis no se encontró literatura científica que analice los EVS en adventistas de países o comunidades de renta baja y condiciones de pobreza, se concluye que los EVS promovidos por la filosofía adventista, presentan los siguientes desafíos:

- El discurso científico de la salud adventista, especialmente aquellos relacionados con el Adventist Health Study, está fuertemente soportado en la medicina ya que se basa en la evidencia, la epidemiología clásica, la medicina preventiva y la salud pública tradicional bajo enfoques de investigación de corte positivista empírico-analítico, pero carecen de evidencia científica con aproximaciones histórico-hermenéuticas y crítico-sociales, los cuales significan sesgos que van en detrimento de la objetividad pretendida.

- $\quad$ El discurso sobre EVS, en el contexto científico adventista, carece de un

\footnotetext{
${ }^{9}$ Agencias Adventista de Noticias. [Internet] Los adventistas ya son más 16,6 millones en el mundo. Igreja Adventista do Sétimo Dia - Portal Oficial. [Cited 2013, January, 20] ]; [about 2 screens]. Available from: http://adventistas.ec/noticias/UE/2010/10_18_adventistas.php.

${ }^{10}$ Wikipedia. [Internet] c2012; [Cited 2013, January, 20]; [about 2 screens]. Available from: http:// en.wikipedia.org/wiki/Loma Linda, California; San Bernardino County. [Internet]. San Bernardino: c2008. Loma Linda; [Cited 2013, January, 20]; [about 1 screen]. Available from: http://www.co.san-bernardino.ca.us/ sheriff/patrol/Loma Linda.asp; Loma Linda City. [Internet]. Loma Linda. c2012; Beautiful Hill; [Cited 2013, January, 20]; [about 2 screens]. Available from: http://www.lomalinda-ca.gov/asp/Site/OurCity/AboutUs/index.asp
} 
soporte en la teoría crítica de la sociedad que realiza las interpretaciones del fenómeno salud-enfermedad desde aproximaciones más holísticas como las planteadas por la medicina social, la salud colectiva y la epidemiología crítica.

- El enfoque de EVS considera a la educación como factor importante para el cambio de hábitos y conductas de riesgo, orientándose al paradigma de "cajas negras" de la epidemiología clásica, limitando su interpretación a lo conductual, causal y de factor de riesgo altamente desacreditado por las corrientes de la salud colectiva y la epidemiología crítica modernas.

- Se recomienda que los abordajes a la investigación en EVS, desde la perspectiva científica adventista, capitalicen la experiencia y la comprensión del enfoque positivista y desarrollen procesos de investigación desde enfoques más holísticos como los que considera la teoría crítica de la sociedad, de tal suerte que permitan comprender y abordar la saludenfermedad con orientaciones que ofrezcan interpretaciones con menor sesgo.

- La conducta individual, en lo que se refiere a la preferencia de EVS, requiere unas condiciones sociales, políticas, económicas, ambientales, demográficas y epidemiológicas favorables para que sean viables como, por ejemplo, seguridad ciudadana, ambiental y alimentaria, empleo, vivienda, entre otros.

- Los EVS podrían ser una limitación a la hora de realizar educación en salud y prevención de la enfermedad, especialmente en comunidades adventistas de países y regiones pobres, incluso para miembros institucionalizados.

- Los programas de salud adventista pueden revisar las estrategias para la intervención de la pobreza e inequidad que realiza la Agencia Adventista de Desarrollo y Recursos Asistenciales (ADRA) en algunos países de África y Sudamérica.

- $\quad$ Se requiere que la comunidad científica adventista avance en estudios sobre EVS mixtos y triangulados soportados que posibiliten analizar los hábitos de vida y salud de la comunidad, asociados a condiciones de 
pobreza e inequidad, en situaciones de guerra, desplazamiento, desastres y poblaciones en adaptación al cambio climático.

- Generar discusiones de orden académico y científico entre los profesionales de la salud adventista para analizar los patrones de morbilidad, mortalidad, padecimiento, discapacidad a partir de los elementos que ofrece la epidemiología social, más allá del énfasis biologista y morbicéntrico que propone la epidemiología clásica. La discusión con profesionales en ciencias sociales y del comportamiento posibilitaría retroalimentar el enfoque. 


\section{Referencias}

Albert, C., Gaziano, J., Willett, C., Manson, E. (2002). Nut Consumption and Decreased Risk of Sudden Cardiac Death in the Physicians' Health Study. Arch Intern Med. 162(12), 1382-1387.

Alcaldía de Medellín. (s/f). Promoción de estilos de vida saludable. Disponible en: http:// www.medellin.gov.co/irj/portal/ciudadanos?NavigationTarget=navext $\% 28 \mathrm{ROL}$ ES://portal_content/MunicipioMedellin/PortalServiciosEnLinea/superAdmin/ roles/slmdm.ep.pcd.serviciosAnonimos/pcm.ep.pcd.temasCiudadano/ salud/wpcnavigation:///wpccontent/Sites/Su.

Álvarez, L. (2012). Los estilos de vida en salud: del individuo al contexto. Rev. Fac. Nac. Salud Pública. 2012; 30(1), 95-101.

Álvarez, G., García, A., Bonet, M. (2007). Pautas conceptuales y metodológicas para explicar los determinantes de los niveles de salud en Cuba. Instituto Nacional de Higiene, Epidemiología y Microbiología Rev Cubana Salud Pública, 33(2).

Álvarez, M., Estrada, A. (2008). Inseguridad alimentaria de los hogares colombianos según localización geográfica y algunas condiciones sociodemográficas. Rev. Perspectivas en nutrición humana. enero - junio, 10 (1), 23-36.

Appel, L. (2008). Dietary Patterns and Longevity: Expanding the Blue Zones. Circulation, 118:214215.

Barton, H., Grant, M. (2006). A Health Map for the Local Human Habitat' inJournal of the Royal Society for the Promotion of Health, 126, (6).

Barton, H., Grant, M. (2012). Urban Planning for Healthy Cities: A Review of the Progress of the European Healthy Cities Programme. To be published in Journal of Urban Health.

Beeson, W., Mills, P., Phillips, R., Andress, M., Fraser, G. (1989). Chronic Disease Among Seventhday Adventists, a low-risk group. Rationale, methodology, and description of the population. Cancer. Aug 1;64(3), 570-81.

Beeson, L. (1999). The Adventist advantage. Revista College and University Dialogue; 11(2), 8-11.

Berkel, J., de Waard, F. (1983). Mortality and Life Expectancy of Seventh-day Adventists in the Netherlands. Int J Epidemiol; 12, 455-459.

Bosch, O. (2006). De Flexner a la educación médica orientada a resultados. II Congreso de la Sociedad de Educación Médica de Euskadi. Bilbao. Universidad del País Vasco. http:// www.ehu.es/SEMDE/II_congreso/PDFs/01_SEMDE_II.pdf

Breilh, J. (2003). Epidemiología crítica. Ciencia emancipadora e interculturalidad. Buenos Aires: Lugar Editorial. 
Buettner, D. (2005). Longevidad: los secretos para una larga vida. Revista National Geographic. Noviembre.

Buettner, D. (2008). The Blue Zone: Lessons For Living Longer from the People Who've Lived the Longest. Washington, DC: National Geographic Society.

Cerecero, P., Hernández, B., Aguirre, D., Valdés, R., Huitrón, G. (2009). Estilos de vida asociados al riesgo cardiovascular global en trabajadores universitarios del Estado de México. Salud Pública; 51(6), 465-473.

Cockerham, W. (2012). Medical Sociology. 12th ed. Boston: Prentice Hall.

Crawford, R. (1977). You are Dangerous to Your Health: the Ideology and Politics of Victim Blaming. The International Journal of Health Services, 7 (4), 663 - 689.

Dahlgren, G., Whitehead, M. (1991). Policies and Strategies to Promote Social Equity in Health. Stockholm Institute for Future Studies.

De la Antonia, L. (2009). Hacia el liderazgo europeo en las escuelas de pensamiento de marketing. 2a. Ed. Madrid: Visión Libros.

De Almeida, N. (2000). La ciencia tímida. Ensayos de deconstrucción de la epidemiología. Buenos Aires: Lugar Editorial.

Dever, A., (1976). An Epidemiological Model for Health Policy Analysis. Social Indicators Research 2, 453-466.

Espinoza, L. (2004). Cambios del modo y estilo de vida; su influencia en el proceso saludenfermedad. Rev Cubana Estomatol, 41(3).

Fernández, J., Orrego, R., Martínez, C., Uribe, L., Quintero, M., Agudelo, V., Franco, J. (2012). Estudio para determinar el nivel de satisfacción de la población beneficiada de actividad física y estilos de vida saludables ofrecidos por la Secretaría de Recreación y el Deporte del Municipio de Itagüí. Universidad Cooperativa de Colombia, 122.

Fonnebo, V., Helseth, A. (1987). A. Cancer Incidence in Norwegian Seventh-day Adventist 1961 to 1986. Cancer 68, 666-671.

Fraser, G. (2003). Diet, Life Expectancy and Chronic Disease. New York, NY: Oxford University Press.

Frenk, J, Londoño, J. (1998). El costo de no reformar. Revista Cuestión Social (México), № 42.

Friis, R., Seller, T. (2009). Epidemiology for Public Health Practice. 4th (ed). Ontario: Jones and Bartlett Publisher. Chapter 4, Descriptive epidemiology: person, place, time, 141 201.

Fuentes, S. (2012). Estilo de vida saludable y teología. Tiempo de elección, tiempo de decisiones. Revista Estilo de vida saludable. (1) 4-6. http://adventistaslascondes.cl/revista_ salud/ESTILOS_DE_VIDA_SALUDABLE_1ra_E_2012.pdf. 


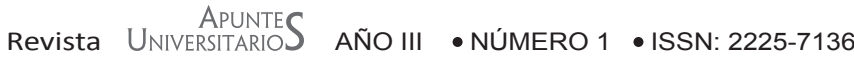

Galvéz, C. (2002). Poder para cambiar los hábitos de salud de manera feliz y para siempre. Lima: Editorial Imprenta Unión.

Gómez, R. (2002). La noción de "salud pública": consecuencias de la polisemia. Rev. Facultad Nacional de Salud Pública. 20 (1), 101-116. Revista Estilo de vida saludable. (1) 7-9. http://adventistaslascondes.cl/revista_salud/ESTILOS_DE_VIDA_SALUDABLE_1ra_E_2012. pdf

Guerrero, R., León, A. (2010). Estilo de vida y salud: un problema socioeducativo. Antecedentes. Revista Venz. De Educ. Educere jun - dic, 14 (9), 287 - 395.

Guerrero, R., León, A. (2010). Estilo de vida y salud. Rev. Venz. De Educ. Educere enero -junio, 14 (48), $13-19$.

Hall, D. (2010). The Vegetarian Advantage. Nampa: Pacific Press Publishing Association.

Iglesia Adventista del Séptimo día. Creencias de los adventistas del séptimo día. 2a (ed). Silver Spring: Pacific Press Publishing Assocation. Capítulo 22, La conducta cristiana, 312329.

Jarillo, S., Guinsberg, E. (eds) (2007). Temas y desafío en salud colectiva. La necesidad de un nuevo paradigma en el campo de la alimentación y la nutrición. Buenos Aires: Lugar Editorial, $127-152$.

Jenson, O. (1993). Cancer Risk Among Danish Male Seventh-day Adventists and Other Temperance Society Members. J Natl Cancer Inst. 12, 455-459.

Jiménez, D. (2012). Estilo de vida saludable y medicina. Rev. Estilo de Vida Saludable. (1) 7-9. http:// adventistaslascondes.cl/revista_salud/ESTILOS_DE_VIDA_SALUDABLE_1ra_E_2012.pdf

Keyeux, G. (2007). Bioética y salud pública: encuentros y tensiones. 1ft (ed). Bogotá D.C.: National Graphics Ltda.

Kuratsune, M., Ikeda, M., Hayashi, T. (1986). Epidemiologic Studies on Possible Health Effects of Intake of Pyrolysates of Foods, with Reference to Mortality Among Japanese Seventhday Adventists. Environ Health Perspect; 67, 143-146.

Lalonde, M. (1974). A New Perspective on the Health of Canadians. A working document. Ottawa: Ministry of National Health and Welfare.

Martínez, J. (2002). Obesidad. Anales del sistema sanitario de Navarra. Serie en Internet. Vol. 25, Sup. 1. Disponible en: http://www.cfnavarra.es/salud/anales/textos/suple25_1.html.

Menéndez, E. (1998). Estilos de vida, riesgos y construcción social. Conceptos similares y significados diferentes. Estudios sociológicos. 16(46), 37-67.

Mills,P., Beeson, W., Phillips, R., Fraser. (1989). Cohort Study of Diet, Lifestyle, and Prostate Cancer in Adventist men. Cancer. Aug 1;64(3), 598-604. 
Nieman, D. (1992). Los adventistas y la salud. Cambio de actitud y conducta respecto a la salud; Bogotá D. C.: Capítulo 4, 40-46.

Organización Mundial de la Salud. (2010). Health Behaviour in School-aged Children. Disponible en: http://www.hbsc.es/pdf/form/tema2.pdf. Consultado 2012 dic 08.

Pamplona, R. (2004). iDisfrútalo! Alimentos que curan y previenen. Madrid: Serie Nuevo Estilo de Vida. Editorial Safeliz, 182.

Schneider, E. (2004). Salud por la naturaleza Tomo I.2a ed. Madrid: Serie Nuevo Estilo de Vida. Editorial Safeliz, 298.

Simon, E., Del Barrio, A. (2002). Leptina y obesidad. Anales del Sistema Sanitario de Navarra. Vol. 25, Sup. 1. Disponible en: http://www.cfnavarra.es/salud/anales/textos/suple25_1.html.

Vargas, S. (2010). Los estilos de vida en la salud. La salud pública en Costa Rica. Estado actual, retos y perspectivas. San José: Universidad de Costa Rica, 539.

White, E. (2010). Ministerio de curación. Miami: Inter-American Division Publishing Association, 372.

World Health Organization. (2010). A Conceptual Framework for Action on the Social Determinants of Health. Social Determinants of Health Discussion Paper 2.Debates, policy \& practice, CASE STUDIES Geneve. 